\title{
A NEARLY-OPTIMAL ALGORITHM FOR THE FREDHOLM PROBLEM OF THE SECOND KIND OVER A NON-TENSOR PRODUCT SOBOLEV SPACE
}

\author{
A.G. WERSCHULZ AND H. WOŹNIAKOWSKI
}

Communicated by Ian Sloan

\begin{abstract}
In a previous paper, the authors showed that the information complexity of the Fredholm problem of the second kind is essentially the same as that of the approximation problems over the spaces of kernels and right-hand sides. This allowed us to give necessary and sufficient conditions for the Fredholm problem to exhibit a particular level of tractability (for information complexity) over weighted tensor product (WTP) spaces, as well as over an important class of not necessarily tensor product weighted Sobolev spaces. Furthermore, we addressed the overall complexity of this Fredholm problem for the case in which the kernels and right-hand sides belong to a WTP space. For this case, we showed that a nearly-minimal-error interpolatory algorithm is easily implementable, with cost very close (to within a logarithmic factor) to the information cost. As a result, tractability results, which had previously only held for the information complexity, now hold for the overall complexity-provided that our kernels and right-hand sides belong to WTP spaces. This result does not hold for the weighted Sobolev spaces mentioned above, since they are not necessarily tensor product spaces.

In this paper, we close this gap. We exhibit an easily implementable iterative approximation to a nearly minimal error interpolatory algorithm for this family of weighted Sobolev spaces. This algorithm exhibits the same good properties as the algorithm presented in the previous paper.
\end{abstract}

2010 AMS Mathematics subject classification. Primary 65R20, Secondary 41A63, 68Q25.

Keywords and phrases. Fredholm problem of the second kind, tractability, information-based complexity.

The second author was supported in part by the National Science Foundation and by the National Science Centre, Poland, based on the decision DEC2013/09/B/ST1/04275.

Received by the editors on April 29, 2014. 
1. Introduction. Let $I=[0,1]$ be the unit interval, and let $d \in \mathbb{N}=$ $\{1,2, \ldots\}$ be a positive integer. For $q \in L_{2}\left(I^{2 d}\right)$ and $f \in L_{2}\left(I^{d}\right)$, we wish to approximate the solution $u \in L_{2}\left(I^{d}\right)$ of the Fredholm problem of the second kind having kernel $q$ and right-hand side $f$, i.e.,

(1) $u(\boldsymbol{x})-\int_{I^{d}} q(\boldsymbol{x}, \boldsymbol{y}) u(\boldsymbol{y}) d \boldsymbol{y}=f(\boldsymbol{x}) \quad$ almost everywhere $\boldsymbol{x} \in I^{d}$.

We rephrase this problem as an operator equation in the usual way. For $q \in L_{2}\left(I^{2 d}\right)$, we let $T_{q}$ be the compact Fredholm operator on $L_{2}\left(I^{d}\right)$ defined by

$$
T_{q} v=\int_{I^{d}} q(\cdot, \boldsymbol{y}) v(\boldsymbol{y}) d \boldsymbol{y} \quad \text { for all } v \in L_{2}\left(I^{d}\right),
$$

so that we may rewrite (1) as

$$
\left(I-T_{q}\right) u=f .
$$

Clearly,

$$
\begin{aligned}
& \left\|T_{q} v\right\|_{L_{2}\left(I^{d}\right)} \leq\|q\|_{L_{2}\left(I^{2 d}\right)}\|v\|_{L_{2}\left(I^{d}\right)} \\
& \text { for all } q \in L_{2}\left(I^{2 d}\right), \quad v \in L_{2}\left(I^{d}\right),
\end{aligned}
$$

so that

$$
\left\|T_{q}\right\|_{\operatorname{Lin}\left[L_{2}\left(I^{d}\right)\right]} \leq\|q\|_{L_{2}\left(I^{2 d}\right)} \text { for all } q \in L_{2}\left(I^{2 d}\right) .
$$

Moreover, if $\|q\|_{L_{2}\left(I^{2 d}\right)}<1$, then the operator $I-T_{q}$ has a bounded inverse, whose operator norm satisfies the inequality

$$
\left\|\left(I-T_{q}\right)^{-1}\right\|_{\operatorname{Lin}\left[L_{2}\left(I^{d}\right)\right]} \leq \frac{1}{1-\|q\|_{L_{2}\left(I^{2 d}\right)}} .
$$

Hence, (2) has a unique solution $u \in L_{2}\left(I^{d}\right)$.

Now suppose that $F_{d}$ and $Q_{2 d}$ are subspaces of $L_{2}\left(I^{d}\right)$ and $L_{2}\left(I^{2 d}\right)$, respectively. Without essential loss of generality we may assume that

$$
\|\cdot\|_{L_{2}\left(I^{d}\right)} \leq\|\cdot\|_{F_{d}} \text { and }\|\cdot\|_{L_{2}\left(I^{2 d}\right)} \leq\|\cdot\|_{Q_{2 d}},
$$

see [2, Remark 3.1]. Given $M \in(0,1)$, let

$$
Q_{2 d}^{\mathrm{res}}=\left\{q \in Q_{2 d}:\|q\|_{Q_{2 d}} \leq M\right\}
$$


Letting $B F_{d}$ denote the unit ball of $F_{d}$, the solution operator $S_{d}: B F_{d}$ $\times Q_{2 d}^{\mathrm{res}} \rightarrow L_{2}\left(I^{d}\right)$ given by

$$
\begin{gathered}
u=S_{d}(f, q) \quad \text { if and only if }\left(I-T_{q}\right) u=f \\
\text { for all }(f, q) \in B F_{d} \times Q_{2 d}^{\mathrm{res}}
\end{gathered}
$$

is well-defined.

We wish to approximate $S_{d}$ at minimal cost. For $(f, q) \in B F_{d} \times Q_{2 d}^{\text {res }}$, we approximate $S_{d}(f, q)$ by an algorithm $A$ using finitely many information evaluations of $f$ and $q$. Here, an information operation is the evaluation of a continuous linear functional (of either the kernel or the right-hand side). ${ }^{1}$

The error of such an algorithm $A$ is given by

$$
e\left(A, S_{d}\right)=\sup _{(f, q) \in B F_{d} \times Q_{2 d}^{\mathrm{res}}}\left\|S_{d}(f, q)-A(f, q)\right\|_{L_{2}\left(I^{d}\right)} .
$$

Then

$$
e\left(n, S_{d}\right)=\inf _{A_{n}} e\left(A_{n}, S_{d}\right)
$$

denotes the $n$th minimal error for solving the Fredholm problem. Here, the infimum is over all algorithms $A_{n}$ using at most $n$ information operations.

For $\varepsilon \in(0,1)$, the information complexity of our problem is given by

$$
n\left(\varepsilon, S_{d}\right)=\inf \left\{n \in \mathbb{N}: e\left(n, S_{d}\right) \leq \varepsilon\right\} .
$$

Remark 1.1. The Fredholm problem (2) is a linear operator equation since the solution $u$ depends linearly on the right hand side $f$. However, our viewpoint is that the solution $u$ depends not only on $f$, but also on the kernel $q$, with algorithms approximating $u$ requiring information about both $f$ and $q$. Since $u$ depends nonlinearly on $q$, we are looking at a nonlinear problem, at least from the viewpoint of information-based complexity.

In our previous paper [2], we examined this problem from the viewpoint of tractability, i.e., the behavior of the problem's $\varepsilon$-complexity as a function of both the error threshold $\varepsilon$ and the dimension $d$, where $d$ is large. We established the following results, which we express in terms of $F=\left\{F_{d}\right\}_{d \in \mathbb{N}}$ and $Q=\left\{Q_{2 d}\right\}_{d \in \mathbb{N}}$ : 
(i) The Fredholm problem is no harder than the $L_{2}$-approximation problems for $F$ and $Q .^{2}$

(ii) The $L_{2}$-approximation problem for $F$ is no harder than the Fredholm problem.

(iii) If $Q$ satisfies a certain extension property, then the $L_{2}$-approximation problem for $Q$ is no harder than the Fredholm problem.

It then follows that, if the $L_{2}$-approximation problems for $F$ and $Q$ enjoy any specific kind of tractability (e.g., weak, polynomial, or strong polynomial, see [2] or [1, subsection 4.4] for details), then so does the Fredholm problem. Moreover, suppose that $Q$ satisfies the extension property; if the $L_{2}$-approximation problem for either $Q$ or $F$ does not enjoy a particular kind of tractability, then neither does the Fredholm problem.

We then used these results, along with results (some known, some new) about the $L_{2}$-approximation problem for certain well-studied spaces: spaces of $C^{\infty}$ functions, (unweighted) tensor product spaces, weighted tensor product (WTP) spaces and a class of not necessarily tensor product weighted Sobolev spaces. We found necessary and sufficient conditions for the Fredholm problem to have various kinds of tractability over these spaces, at least at the level of information complexity. Once again, see our paper [2] for the details.

But information complexity does not tell us the whole story, since it counts only the number of information functionals evaluated, ignoring the combinatory cost (e.g., arithmetic and boolean operations) of the algorithm that computes the approximate solution. We really want to determine the (overall) complexity of our problem, given by

$$
\operatorname{comp}\left(\varepsilon, S_{d}\right)=\inf \left\{\operatorname{cost}(A): \text { algorithms } A \text { such that } e\left(A, S_{d}\right) \leq \varepsilon\right\} .
$$

Here, $\operatorname{cost}(A)$ denotes the cost of an algorithm $A$, including both the information and the combinatory costs.

This point was addressed in the last part of [2]. Under the condition that $F$ and $Q$ were sequences of WTP spaces, we showed that an iterative approximation to the interpolatory algorithm delivers an $\varepsilon$ approximation at nearly-minimal cost. By "nearly minimal," we mean that its cost is at most a factor of $\ln (1 / \varepsilon)$ more than the optimal cost.

The last result does not hold for the weighted Sobolev spaces mentioned previously since they are not necessarily WTP spaces. So, 
although we have conditions that are necessary and sufficient for various kinds of tractability when using such spaces, these tractability results only apply at the information level. The question of whether the same is true for overall complexity (i.e., counting combinatory cost, as well as information cost) was not settled by [2].

In this paper, we close this gap. Let $F_{d}$ and $Q_{2 d}$ be the weighted Sobolev spaces mentioned above. From [2], we know that an interpolatory algorithm has nearly-minimal error. Our main result is to exhibit an iterative approximation of this algorithm, whose error is nearly minimal and whose cost is about the same, modulo a logarithmic factor. It then follows that all the statements about the tractability (at the information level) of the Fredholm problem for this family of weighted Sobolev spaces also hold when combinatory cost is included.

In general, these Sobolev spaces are not tensor product spaces. That is because the eigenvalues of the associated operator defined by (6) in Section 2 are not generally of product form. Hence, the results of [2] do not directly apply here. However, the eigenvectors of this operator do have product form. This allows us to prove that the corresponding matrix of linear equations is sparse and the cost of one step of an iterative algorithm is linear, modulo a logarithmic factor, in the size of this matrix.

2. A family of weighted Sobolev spaces. We now define the family of weighted Sobolev spaces studied in this paper. These spaces are weighted subspaces of $\left[H^{1}(I)\right]^{\otimes d}$, the $d$-fold tensor product of $H^{1}(I)$.

Let

$$
\gamma=\left\{\gamma_{d, \mathfrak{u}}: \mathfrak{u} \subseteq[d], d \in \mathbb{N}\right\}
$$

be a set of non-negative weights, where $[d]=\{1,2, \ldots, d\}$. For simplicity, we shall choose $\gamma_{d, \emptyset}=1$ and assume that all the weights are in the interval $[0,1]$. Let

$$
H_{d, \boldsymbol{\gamma}}=\left\{v \in\left[H^{1}(I)\right]^{\otimes d}: \text { for all } \mathfrak{u} \subseteq[d] \gamma_{d, \mathfrak{u}}=0 \Longrightarrow \frac{\partial^{|\mathfrak{u}|}}{\partial x_{\mathfrak{u}}} v \equiv 0\right\}
$$

which is a Hilbert space under the inner product

$$
\langle v, w\rangle_{H_{d, \gamma}}=\int_{I^{d}} v(\boldsymbol{x}) w(\boldsymbol{x}) d \boldsymbol{x}
$$




$$
\begin{aligned}
& +\sum_{\substack{\mathfrak{u} \subseteq[d] \\
\mathfrak{u} \neq \emptyset \\
\gamma_{d, \mathfrak{u}}>0}} \gamma_{d, \mathfrak{u}}{ }^{-1} \int_{I^{d}} \frac{\partial^{|\mathfrak{u}|}}{\partial x_{\mathfrak{u}}} v(\boldsymbol{x}) \frac{\partial^{|\mathfrak{u}|}}{\partial x_{\mathfrak{u}}} w(\boldsymbol{x}) d \boldsymbol{x} \\
& \quad \text { for all } v, w \in H_{d, \boldsymbol{\gamma}} .
\end{aligned}
$$

Interpreting $0 / 0$ as 0 , we may rewrite this inner product in the simpler form

$$
\begin{gathered}
\langle v, w\rangle_{H_{d, \boldsymbol{\gamma}}}=\sum_{\mathfrak{u} \subseteq[d]} \gamma_{d, \mathfrak{u}}{ }^{-1} \int_{I^{d}} \frac{\partial^{|\mathfrak{u}|}}{\partial x_{\mathfrak{u}}} v(\boldsymbol{x}) \frac{\partial^{|\mathfrak{u}|}}{\partial x_{\mathfrak{u}}} w(\boldsymbol{x}) d \boldsymbol{x}, \\
\text { for all } v, w \in H_{d, \boldsymbol{\gamma}} .
\end{gathered}
$$

Note that, if $\gamma_{d, \mathfrak{u}}=0$ for all nonempty $\mathfrak{u}$, then $H_{d, \gamma}=\operatorname{span}\{1\}$. To omit this trivial case, we assume that $\gamma_{d, \mathfrak{u}} \neq 0$ for at least one nonempty $\mathfrak{u}$. Then it is easy to see that $\operatorname{dim}\left(H_{d, \gamma}\right)=\infty$.

It is natural to ask how the linear spaces $H_{d, \boldsymbol{\gamma}}$ and $\left[H^{1}(I)\right]^{\otimes d}$ are related. Obviously, we always have $H_{d, \boldsymbol{\gamma}} \subseteq\left[H^{1}(I)\right]^{\otimes d}$. If $\gamma_{d, \mathfrak{u}}=0$ for some nonempty $\mathfrak{u}$, then $H_{d, \boldsymbol{\gamma}}$ is a proper subspace of $\left[H^{1}(I)\right]^{\otimes d}$. Otherwise, if all the $\gamma_{d, \mathfrak{u}}$ are positive, then $H_{d, \gamma}=\left[H^{1}(I)\right]^{\otimes d}$; however, their inner products and norms are different unless all $\gamma_{d, \mathfrak{u}}=1$.

The space $\left[H^{1}(I)\right]^{\otimes d}$ is a tensor-product space. How about the space $H_{d, \boldsymbol{\gamma}}$ ? The answer depends on the weights $\boldsymbol{\gamma}$. Indeed, it is easy to see that $H_{d, \boldsymbol{\gamma}}$ is a tensor product space for the so-called positive product weights, i.e., for $\gamma_{d, \mathfrak{u}}=\prod_{j \in \mathfrak{u}} \gamma_{d, j}$ with $\gamma_{d, j} \in(0,1]$. Then $H_{d, \boldsymbol{\gamma}}=\bigotimes_{j=1}^{d} H_{1, \gamma_{d, j}}(I)$, where $H_{1, \gamma_{d, j}}$ is algebraically the same as $H^{1}(I)$, but with inner product

$$
\begin{aligned}
\langle u, w\rangle_{H_{1, \gamma_{d, j}}(I)}= & \int_{0}^{1} v(x) w(x) d x+\gamma_{d, j}^{-1} \int_{0}^{1} v^{\prime}(x) w^{\prime}(x) d x \\
& \text { for all } v, w \in H^{1}(I) .
\end{aligned}
$$

To see that $H_{d, \gamma}$ is not always a tensor-product space, consider the following example. Choose $\gamma_{d, \mathfrak{u}} \neq 0$ for all $|\mathfrak{u}|=1$ and $\gamma_{d, \mathfrak{u}}=0$ for all $|\mathfrak{u}| \geq 2$. Then $H_{d, \gamma}$ is the direct sum of univariate functions and, for $f \in H_{d, \boldsymbol{\gamma}}$, we have

$$
f(\boldsymbol{x})=f_{1}\left(x_{1}\right)+f_{2}\left(x_{2}\right)+\cdots+f_{d}\left(x_{d}\right) \quad \text { with } \quad f_{j} \in H^{1}(I),
$$


and

$$
\|f\|_{H_{d, \gamma}}^{2}=\|f\|_{L_{2}\left(I^{d}\right)}^{2}+\sum_{j=1}^{d} \gamma_{d,\{j\}}^{-1}\left\|f_{j}^{\prime}\right\|_{L_{2}(I)}^{2} .
$$

In particular, $f(\boldsymbol{x})=\prod_{j=1}^{d} f_{j}\left(x_{j}\right)$ does not belong to $H_{d, \boldsymbol{\gamma}}$ for nonconstant $f_{j} \in H^{1}(I)$, which shows that $H_{d, \gamma}$ is not a tensor product space in this case.

We will need to know the eigenvalues and eigenfunctions of the operator

$$
W_{d, \boldsymbol{\gamma}}=E_{d, \boldsymbol{\gamma}}{ }^{*} E_{d, \boldsymbol{\gamma}}: H_{d, \boldsymbol{\gamma}} \longrightarrow H_{d, \boldsymbol{\gamma}}
$$

where $E_{d, \boldsymbol{\gamma}}: H_{d, \boldsymbol{\gamma}} \rightarrow L_{2}\left(I^{d}\right)$ is the canonical embedding

$$
E_{d, \boldsymbol{\gamma}} v=v, \quad \text { for all } v \in H_{d, \boldsymbol{\gamma}} .
$$

This corresponds to the $L_{2}$-approximation problem for $H_{d, \boldsymbol{\gamma}}$ and its $n$th minimal error is given by

$$
e\left(n, E_{d, \boldsymbol{\gamma}}\right)=\sqrt{\alpha_{d, \boldsymbol{\gamma}, n+1}},
$$

where

$$
\alpha_{d, \boldsymbol{\gamma}, 1} \geq \alpha_{d, \boldsymbol{\gamma}, 2} \geq \alpha_{d, \boldsymbol{\gamma}, 3} \cdots>0
$$

are the eigenvalues of $W_{d, \boldsymbol{\gamma}}$. Moreover, the corresponding $n$th minimalerror algorithm is given by the projection

$$
P_{d, \boldsymbol{\gamma}, n} w=\sum_{j=1}^{n} \frac{\left\langle w, e_{d, \boldsymbol{\gamma}, j}\right\rangle_{H_{d, \boldsymbol{\gamma}}}}{\left\|e_{d, \boldsymbol{\gamma}, j}\right\|_{H_{d, \boldsymbol{\gamma}}}^{2}} e_{d, \boldsymbol{\gamma}, j} \quad \text { for all } w \in H_{d, \boldsymbol{\gamma}}
$$

where $e_{d, \boldsymbol{\gamma}, j}$ is the eigenfunction of $W_{d, \boldsymbol{\gamma}}$ corresponding to $\alpha_{d, \boldsymbol{\gamma}, j}$. The set of eigenfunctions $\left\{e_{d, \boldsymbol{\gamma}, j}\right\}_{j \in \mathbb{N}}$ is an orthogonal basis for $H_{d, \boldsymbol{\gamma}}$.

So let us determine these eigenvalues and eigenfunctions of $W_{d, \boldsymbol{\gamma}}$. For $d \in \mathbb{N}$ and any multi-index $\mathbf{k}=\left[k_{1}, k_{2}, \ldots, k_{d}\right] \in \mathbb{N}^{d}$, let

$$
e_{\mathbf{k}}=\bigotimes_{i=1}^{d} e_{k_{i}}, \quad \text { i.e., } e_{\mathbf{k}}(\boldsymbol{x})=\prod_{i=1}^{d} e_{k_{i}}\left(x_{i}\right) \quad \text { for all } x \in I^{d}
$$

where

$$
e_{k}(\boldsymbol{x})=\cos \pi(k-1) x, \quad \text { for all } x \in I, k \in \mathbb{N} \text {. }
$$


Note that

$$
\left\|e_{\mathbf{k}}\right\|_{L_{2}\left(I^{d}\right)}^{2}=2^{-\left|\left\{j \in[d]: k_{j}>1\right\}\right|}, \quad \text { for all } \mathbf{k} \in \mathbb{N}^{d} .
$$

We now check when $e_{\mathbf{k}}$ belongs to $H_{d, \boldsymbol{\gamma}}$. It clearly holds if all the $\gamma_{d, \mathfrak{u}}$ are positive. On the other hand, if $\gamma_{d, \mathfrak{u}}=0$ for some $\mathfrak{u}$, then we need to guarantee that $\partial^{|\mathfrak{u}|} / \partial x_{\mathfrak{u}} e_{\mathbf{k}} \equiv 0$. This holds if and only if there is an index $j \in \mathfrak{u}$ for which $k_{j}=0$. Hence, $e_{\mathbf{k}} \in H_{d, \boldsymbol{\gamma}}$ if and only if $\mathbf{k} \in J_{d, \boldsymbol{\gamma}}$, where

$$
J_{d, \boldsymbol{\gamma}}=\left\{\mathbf{k} \in \mathbb{N}^{d}: \forall \mathfrak{u} \subseteq[d], \gamma_{d, \mathfrak{u}}=0 \Longrightarrow \exists j \in \mathfrak{u} \text { such that } k_{j}=1\right\} .
$$

We stress that $J_{d, \boldsymbol{\gamma}}$ is an infinite set since $\gamma_{d, \mathfrak{u}} \neq 0$ for at least one nonempty $\mathfrak{u}$. Clearly, $J_{d, \boldsymbol{\gamma}}=\mathbb{N}^{d}$ if all $\gamma_{d, \mathfrak{u}}$ are positive.

We find that:

$$
W_{d, \boldsymbol{\gamma}} e_{\mathbf{k}}=\alpha_{d, \boldsymbol{\gamma}, \mathbf{k}} e_{\mathbf{k}} \quad \text { for all } \mathbf{k} \in J_{d, \boldsymbol{\gamma}}
$$

where

$$
\alpha_{d, \boldsymbol{\gamma}, \mathbf{k}}=\left(\sum_{\mathfrak{u} \subseteq[d]} \gamma_{d, \mathfrak{u}}{ }^{-1} \prod_{j \in \mathfrak{u}}\left(\pi^{2}\left(k_{j}-1\right)^{2}\right)\right)^{-1} \quad \text { for all } \mathbf{k} \in J_{d, \boldsymbol{\gamma}} .
$$

Then $\left\{e_{\mathbf{k}}\right\}_{\mathbf{k} \in J_{d, \boldsymbol{\gamma}}}$ is an orthogonal basis for $H_{d, \boldsymbol{\gamma}}$, with

$$
\left\|e_{\mathbf{k}}\right\|_{H_{d, \gamma}}^{2}=\frac{\left\|e_{\mathbf{k}}\right\|_{L_{2}\left(I^{d}\right)}^{2}}{\alpha_{d, \boldsymbol{\gamma}, \mathbf{k}}} \quad \text { for all } \mathbf{k} \in J_{d, \boldsymbol{\gamma}}
$$

We stress that the eigenfunctions $e_{\mathbf{k}}$ are of product form and do not depend on the weights $\gamma$. It turns out that this property will be useful later. On the other hand, the eigenvalues $\alpha_{d, \boldsymbol{\gamma}, \mathbf{k}} d o$ depend on the weights $\gamma$ and they are not necessarily of product form.

Now let $\{\mathbf{k}[d, \boldsymbol{\gamma}, j]\}_{j \in \mathbb{N}}$ be a reordering of $J_{d, \boldsymbol{\gamma}}$ such that the $j$ thlargest eigenvalue of $W_{d, \boldsymbol{\gamma}}$ is given by

$$
\alpha_{d, \boldsymbol{\gamma}, j}=\alpha_{\mathbf{k}[d, \boldsymbol{\gamma}, j]} .
$$

We then denote

$$
e_{d, \boldsymbol{\gamma}, j}=e_{\mathbf{k}[d, \boldsymbol{\gamma}, j]}, \quad \text { for all } j \in \mathbb{N},
$$

thereby completing the specification of the $n$th minimal-error algorithm (8) for $H_{d, \boldsymbol{\gamma}}$. 
In what follows, we let $F_{d}=H_{d, \gamma_{F}}$ and $Q_{2 d}=H_{2 d, \gamma_{Q}}$. Here, the weights

$$
\gamma_{F}=\left\{\gamma_{d, \mathfrak{u}, F}: \mathfrak{u} \subseteq[d], d \in \mathbb{N}\right\}
$$

and

$$
\gamma_{Q}=\left\{\gamma_{2 d, \mathfrak{u}, Q}: \mathfrak{u} \subseteq[2 d], d \in \mathbb{N}\right\}
$$

are, in general, not related. However, as before, we assume that $\gamma_{d, \emptyset, F}=\gamma_{2 d, \emptyset, Q}=1, \gamma_{d, \mathfrak{u}, F}, \gamma_{2 d, \mathfrak{u}, Q} \in[0,1]$, and at least one $\gamma_{d, \mathfrak{u}, F}$ and $\gamma_{2 d, \mathfrak{u}, Q}$ are nonzero for a nonempty $\mathfrak{u}$.

3. An interpolatory algorithm for the Fredholm problem. The basic interpolatory algorithm for the Fredholm problem over the Sobolev spaces described above is the same as the interpolatory algorithm described in [2, Section 6]. For the sake of exposition, we repeat that description in this paper.

Let $n(F)$ and $n(Q)$ be two positive integers. The information about any right-hand side $f \in F_{d}$ will be given by the first $n(F)$ inner products with respect to $\left\{e_{d, \gamma_{F}, j}\right\}_{j \in \mathbb{N}}$, and the information about any kernel function $q \in Q_{2 d}$ will be given by the first $n(Q)$ inner products with respect to $\left\{e_{2 d, \gamma_{Q}, j}\right\}_{j \in \mathbb{N}}$. Knowing this information, we can compute

$$
\widetilde{f}=P_{d, \gamma_{F}, n(F)} f \quad \text { and } \quad \widetilde{q}=P_{2 d, \gamma_{Q}, n(Q)} q,
$$

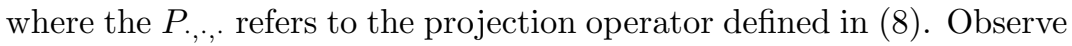
that $(\tilde{f}, \widetilde{q}) \in B F_{d} \times Q_{2 d}^{\text {res }}$. Furthermore, we see that $(\tilde{f}, \widetilde{q})$ interpolate the data, i.e., the first $n(F)$ inner products of $f$ and $\tilde{f}$ are the same, as well as the first $n(Q)$ inner products of $q$ and $\widetilde{q}$.

The interpolatory algorithm is defined as the exact solution of the Fredholm problem for $(\widetilde{f}, \widetilde{q})$, i.e.,

$$
A_{n(F), n(Q)}^{\mathrm{INT}}(f, q)=S_{d}(\tilde{f}, \widetilde{q}) \quad \text { for all }(f, q) \in B F_{d} \times Q_{2 d}^{\mathrm{res}}
$$

Let us choose $n(F)$ and $n(Q)$ as

$$
n(F)=n\left(\frac{1}{2}(1-M) \varepsilon, E_{d, \gamma_{F}}\right)
$$


and

$$
n(Q)=n\left(\frac{(1-M)^{2}}{2 M} \varepsilon, E_{2 d, \gamma_{Q}}\right)
$$

so that

$$
e\left(n(F), E_{d, \gamma_{F}}\right) \leq \frac{1}{2}(1-M) \varepsilon
$$

and

$$
e\left(n(Q), E_{2 d, \gamma_{Q}}\right) \leq \frac{(1-M)^{2}}{2 M} \varepsilon .
$$

We know from $[\mathbf{2}$, Section 6$]$ that

$$
e\left(A_{n(F), n(Q)}^{\mathrm{INT}}, S_{d}\right) \leq \varepsilon,
$$

so we can compute the $\varepsilon$-approximation delivered by $A_{n(F), n(Q)}^{\mathrm{INT}}$ using $n(F)+n(Q)$ information evaluations.

We now look at the implementation of this interpolatory algorithm. The details are similar to those in [2], but with some important differences.

First, note the following crucial (but trivial) observation: For a given $j \in\left\{1,2, \ldots, n_{Q}\right\}$, let us represent $\mathbf{k}\left[2 d, \boldsymbol{\gamma}_{Q}, j\right] \in \mathbb{N}^{2 d}$ as

$$
\mathbf{k}\left[2 d, \gamma_{Q}, j\right]=\left(\mathbf{k}\left[1 ; d, \gamma_{Q}, j\right], \mathbf{k}\left[2 ; d, \gamma_{Q}, j\right]\right),
$$

so that $\mathbf{k}\left[1 ; d, \gamma_{Q}, j\right] \in \mathbb{N}^{d}$ and $\mathbf{k}\left[2 ; d, \boldsymbol{\gamma}_{Q}, j\right] \in \mathbb{N}^{d}$, respectively, denote the first and the last $d$ components of $\mathbf{k}\left[2 d, \gamma_{Q}, j\right]$. Using (9), we then see that

$$
e_{2 d, \gamma_{Q}, j}=e_{\mathbf{k}\left[2 d, \boldsymbol{\gamma}_{Q}, j\right]}=e_{\mathbf{k}\left[1 ; d, \boldsymbol{\gamma}_{Q}, j\right]} \otimes e_{\mathbf{k}\left[2 ; d, \boldsymbol{\gamma}_{Q}, j\right]} .
$$

For $(f, q) \in B F_{d} \times Q_{2 d}^{\mathrm{res}}$, and $\widetilde{u}=A_{n(F), n(Q)}^{\mathrm{INT}}(f, q)$, let

$$
\zeta_{j}=\frac{\left\langle q, e_{2 d, \gamma_{Q}, j}\right\rangle_{H_{2 d, \gamma_{Q}}}}{\left\|e_{2 d, \gamma_{Q}, j}\right\|_{H_{2 d, \gamma_{Q}}}^{2}} \quad \text { for } j \in\{1,2, \ldots, n(Q)\}
$$

and

$$
\theta_{i}=\frac{\left\langle f, e_{d, \boldsymbol{\gamma}_{F}, i}\right\rangle_{H_{d, \boldsymbol{\gamma}_{F}}}}{\left\|e_{d, \boldsymbol{\gamma}_{F},},\right\|_{H_{d, \boldsymbol{\gamma}_{F}}}^{2}} \quad \text { for } i \in\{1,2, \ldots, n(F)\}
$$


Since $A_{n(F), n(Q)}^{\mathrm{INT}}$ is interpolatory, we have

$$
\begin{aligned}
\widetilde{u} & =\int_{I^{d}} \widetilde{q}(\cdot, \boldsymbol{y}) \widetilde{u}(\boldsymbol{y}) d \boldsymbol{y}+\widetilde{f} \\
& =\sum_{j=1}^{n(Q)} \zeta_{j}\left\langle e_{\mathbf{k}\left[2 ; d, \boldsymbol{\gamma}_{Q}, j\right]}, \widetilde{u}\right\rangle_{L_{2}\left(I^{d}\right)} e_{\mathbf{k}\left[1 ; d, \boldsymbol{\gamma}_{Q}, j\right]}+\sum_{i=1}^{n(F)} \theta_{i} e_{\mathbf{k}\left[d, \boldsymbol{\gamma}_{F}, i\right]}
\end{aligned}
$$

This shows that

$$
\begin{aligned}
& \widetilde{u} \in E_{n(F), n(Q)} \\
& =\operatorname{span}\left\{e_{\mathbf{k}\left[d, \boldsymbol{\gamma}_{F}, 1\right]}, \ldots, e_{\mathbf{k}\left[d, \boldsymbol{\gamma}_{F}, n(F)\right]}, e_{\mathbf{k}\left[1 ; d, \boldsymbol{\gamma}_{Q}, 1\right]}, \ldots, e_{\mathbf{k}\left[1 ; d, \boldsymbol{\gamma}_{Q}, n(Q)\right]}\right\} .
\end{aligned}
$$

Now the functions $e_{\mathbf{k}\left[d, \boldsymbol{\gamma}_{F}, i\right]}$ are $L_{2}\left(I^{d}\right)$-orthogonal for $i \in\{1,2, \ldots$, $n(F)\}$, as are the functions $e_{\mathbf{k}\left[1 ; d, \boldsymbol{\gamma}_{Q}, j\right]}$ for $j \in\{1,2, \ldots, n(Q)\}$. They are also mutually $L_{2}\left(I^{d}\right)$-orthogonal if $\mathbf{k}\left[d, \gamma_{f}, i\right] \neq \mathbf{k}\left[1 ; d, \gamma_{Q}, j\right]$, i.e.,

$$
\left\langle e_{\mathbf{k}\left[d, \boldsymbol{\gamma}_{F}, i\right]}, e_{\mathbf{k}\left[1 ; d, \boldsymbol{\gamma}_{Q}, j\right]}\right\rangle_{L_{2}\left(I^{d}\right)}=0
$$

Therefore,

$$
m:=\operatorname{dim} E_{n(F), n(Q)} \in[\max (n(F), n(Q)), n(F)+n(Q)]
$$

The dimension $m=n(F)+p$, where $p$ is the number of indices $\mathbf{k}\left[1 ; d, \gamma_{Q}, j\right]$ that are not present in $\mathbf{k}\left[d, \gamma_{F}, i\right]$ for all $i=1,2, \ldots, n(F)$. Clearly, $p \in[0, n(Q)]$. We remove all redundant $e_{\mathbf{k}\left[1 ; d, \boldsymbol{\gamma}_{Q}, j\right]}$ that belong to $\operatorname{span}\left\{e_{\mathbf{k}\left[d, \boldsymbol{\gamma}_{F}, 1\right]}, \ldots, e_{\mathbf{k}\left[d, \boldsymbol{\gamma}_{F}, n(F)\right]}\right\}$, calling the remaining elements $e_{\mathbf{k}\left[1 ; d, \boldsymbol{\gamma}_{Q}, l_{1}\right]}, \ldots, e_{\mathbf{k}\left[1 ; d, \boldsymbol{\gamma}_{Q}, m-n(F)\right]}$. Let

$$
z_{j}= \begin{cases}e_{\mathbf{k}\left[d, \boldsymbol{\gamma}_{F}, j\right]} & \text { for } j \in\{1,2, \ldots, n(F)\} \\ e_{\mathbf{k}\left[1 ; d, \boldsymbol{\gamma}_{Q}, l_{j-n(F)}\right]} & \text { for } j \in\{n(F)+1, n(F)+2, \ldots, m\} .\end{cases}
$$

Then $\left\{z_{1}, z_{2}, \ldots, z_{m}\right\}$ is an orthogonal basis for $E_{n(F), n(Q)}$. Since $\widetilde{u} \in E_{n(F), n(Q)}$, there exist $v_{1}, v_{2}, \ldots, v_{m} \in \mathbb{R}$ such that

$$
\widetilde{u}=\sum_{k=1}^{m} v_{k} z_{k}
$$


Using this expansion in (10), we see that

(11) $\widetilde{u}=\sum_{k=1}^{m} v_{k} \sum_{j=1}^{n(Q)} \zeta_{j}\left\langle e_{\mathbf{k}\left[2 ; d, \boldsymbol{\gamma}_{Q}, j\right]}, z_{k}\right\rangle_{L_{2}\left(I^{d}\right)} e_{\mathbf{k}\left[1 ; d, \boldsymbol{\gamma}_{Q}, j\right]}+\sum_{j=1}^{n(F)} \theta_{j} e_{\mathbf{k}\left[d, \boldsymbol{\gamma}_{F}, j\right]}$.

Fix $i \in\{1 \ldots, m\}$. Using the $L_{2}\left(I^{d}\right)$-orthogonality of $z_{1}, z_{2}, \ldots, z_{m}$, it follows that

$$
\begin{aligned}
v_{i}= & \frac{\left\langle\widetilde{u}, z_{i}\right\rangle_{L_{2}\left(I^{d}\right)}}{\left\|z_{i}\right\|_{L_{2}\left(I^{d}\right)}^{2}} \\
= & \sum_{k=1}^{m} v_{k} \sum_{j=1}^{n(Q)} \frac{\zeta_{j}\left\langle e_{\mathbf{k}\left[2 ; d, \boldsymbol{\gamma}_{Q}, j\right]}, z_{k}\right\rangle_{L_{2}\left(I^{d}\right)}\left\langle e_{\mathbf{k}\left[1 ; d, \boldsymbol{\gamma}_{Q}, j\right]}, z_{i}\right\rangle_{L_{2}\left(I^{d}\right)}}{\left\|z_{i}\right\|_{L_{2}\left(I^{d}\right)}^{2}} \\
& +\sum_{j=1}^{n(F)} \frac{\theta_{j}\left\langle e_{\mathbf{k}\left[d, \boldsymbol{\gamma}_{F}, j\right]}, z_{i}\right\rangle_{L_{2}\left(I^{d}\right)}}{\left\|z_{i}\right\|_{L_{2}\left(I^{d}\right)}^{2}} .
\end{aligned}
$$

Note that the second sum is $\theta_{i}$ when $i \in\{1, \ldots, n(F)\}$ and zero for $i \in\{n(F)+1, \ldots, m\}$. Letting

$$
\mathbf{u}=\left[v_{1}, v_{2}, \ldots, v_{m}\right]^{\top},
$$

we can write (11) as a system

$$
(\mathbf{I}-\mathbf{K}) \mathbf{u}=\mathbf{b}
$$

of linear equations, where $\mathbf{I}$ denotes the $m \times m$ identity matrix; the $m \times m$ matrix $\mathbf{K}=\left[\kappa_{i, k}\right]_{1 \leq i, k \leq m}$ is given by

$$
\begin{gathered}
\kappa_{i, k}=\sum_{j=1}^{n(Q)} \frac{\zeta_{j}\left\langle e_{\mathbf{k}\left[2 ; d, \boldsymbol{\gamma}_{Q}, j\right]}, z_{k}\right\rangle_{L_{2}\left(I^{d}\right)}\left\langle e_{\mathbf{k}\left[1 ; d, \boldsymbol{\gamma}_{Q}, j\right]}, z_{i}\right\rangle_{L_{2}\left(I^{d}\right)}}{\left\|z_{i}\right\|_{L_{2}\left(I^{d}\right)}^{2}} \\
\text { for } i, k \in\{1,2, \ldots, m\},
\end{gathered}
$$

and

$$
\mathbf{b}=[\theta_{1}, \theta_{2}, \ldots, \theta_{n(F)}, \underbrace{0,0, \ldots, 0}_{m-n(F) \text { times }}] .
$$

Corresponding to [2, Lemma 6.1], we have 
Lemma 3.1. Define

$$
\begin{aligned}
\mathscr{I}=\left\{\mathbf{k}\left[2 d, \gamma_{Q}, j\right]=\left(\mathbf{k}\left[1 ; d, \gamma_{Q}, j\right], \mathbf{k}\left[2 ; d, \gamma_{Q}, j\right]\right):\right. & \\
& j \in\{1,2, \ldots, n(Q)\}\} .
\end{aligned}
$$

(i) For any $i, k \in\{1,2, \ldots, m\}$, we have

$$
\kappa_{i, k}= \begin{cases}\zeta_{j} 2^{-\left|\left\{l \in[d]: k_{l}\left[2 ; d, \boldsymbol{\gamma}_{Q}, j\right]>1\right\}\right|} & \text { if }(i, k)=\left(\mathbf{k}\left[1 ; d, \boldsymbol{\gamma}_{Q}, j\right], \mathbf{k}\left[2 ; d, \boldsymbol{\gamma}_{Q}, j\right]\right) \\ 0 & \text { for some } j \in\{1,2, \ldots, n(Q)\}, \\ & \text { if }(i, j) \notin \mathscr{I},\end{cases}
$$

and so the matrix $\mathbf{K}$ has at most $n(Q)$ non-zero elements.

(ii) $\|\mathbf{K}\|_{\operatorname{Lin}\left[\ell_{2}\left(\mathbb{R}^{m}\right)\right]} \leq M<1$.

(iii) The matrix $\mathbf{I}-\mathbf{K}$ is invertible, with

$$
\left\|(\mathbf{I}-\mathbf{K})^{-1}\right\|_{\operatorname{Lin}\left[\ell_{2}\left(\mathbb{R}^{m}\right)\right]} \leq \frac{1}{1-M} .
$$

Proof. For part (i), note that $\kappa_{i, k}$ is nonzero if and only if there exists $j \in\{1,2, \ldots, n(Q)\}$ such that

$$
z_{i}=e_{\mathbf{k}\left[1 ; d, \gamma_{Q}, j\right]} \quad \text { and } \quad z_{k}=e_{\mathbf{k}[2 ; d, \boldsymbol{\gamma}(Q), j]},
$$

i.e., when $(i, k) \in \mathscr{I}$. When this holds, we have $\kappa_{i, k}=\zeta_{j}\left\|z_{k}\right\|_{L_{2}\left(I^{d}\right)}^{2}=\zeta_{j}\left\|e_{\mathbf{k}\left[2 ; d, \boldsymbol{\gamma}_{Q}, j\right]}\right\|_{L_{2}\left(I^{d}\right)}^{2}=\zeta_{j} 2^{-\left|\left\{l \in[d]: k_{l}\left[2 ; d, \boldsymbol{\gamma}_{Q}, j\right]>1\right\}\right|}$. Obviously, $\kappa_{i, k}=0$ when $(i, j) \notin \mathscr{I}$. Hence, there are at most $|\mathscr{I}|=n(Q)$ nonzero coefficients in the matrix $\mathbf{K}$. This establishes part (i).

To see that part (ii) holds, we estimate $\|\mathbf{K}\|_{\operatorname{Lin}\left[\ell_{2}\left(\mathbb{R}^{n(F)}\right)\right]}^{2}$ by the square of the Frobenius norm $\sum_{i, k=1}^{n(Q)} \kappa_{i, k}^{2}$ and then apply part (i). We have

$$
\begin{aligned}
\|\mathbf{K}\|_{\operatorname{Lin}\left[\ell_{2}\left(\mathbb{R}^{n(F)}\right)\right]}^{2} & \leq \sum_{i, k=1}^{n(Q)} \kappa_{i, k}^{2} \leq \sum_{j=1}^{n(Q)} \zeta_{j}^{2}\left\|e_{\mathbf{k}\left[2 ; d, \boldsymbol{\gamma}_{Q}, j\right]}\right\|_{L_{2}\left(I^{d}\right)}^{2} \\
& \leq \sum_{j=1}^{n(Q)} \zeta_{j}^{2} \leq \sum_{j=1}^{n(Q)} \frac{\left\langle q, e_{2 d, \boldsymbol{\gamma}_{Q}, j}\right\rangle_{H_{2 d, \boldsymbol{\gamma}_{Q}}}}{\left.\| e_{2 d, \boldsymbol{\gamma}_{Q}, j}\right\}_{H_{2 d, \boldsymbol{\gamma}_{Q}}}^{2}} \leq \| M^{2} \leq 1 \\
& =\left\|P_{2 d, \boldsymbol{\gamma}_{Q}, n(Q)} q\right\|_{H_{2 d, \boldsymbol{\gamma}_{Q}}}^{2} \leq\|q\|_{H_{2 d, \boldsymbol{\gamma}_{Q}}} \leq M^{2}
\end{aligned}
$$

as required. Part (iii) follows immediately from part (ii). 
As in [2], we have reduced the implementation of $A_{n(F), n(Q)}^{\mathrm{INT}}$ to solving the sparse linear system $(\mathbf{I}-\mathbf{K}) \mathbf{u}=\mathbf{b}$. We again approximate the solution $\mathbf{u}$ via the simple fixed-point iteration

$$
\begin{aligned}
\mathbf{u}^{(\ell+1)} & =\mathbf{K} \mathbf{u}^{(\ell)}+\mathbf{b} \quad(0 \leq \ell<r), \\
\mathbf{u}^{(0)} & =\mathbf{0} .
\end{aligned}
$$

Letting

$$
\mathbf{u}^{(r)}=\left[v_{1}^{(r)}, v_{2}^{(r)}, \ldots, v_{m}^{(r)}\right]^{\top},
$$

we shall write

$$
u_{n(F), n(Q)}^{(r)}=\sum_{k=1}^{m} v_{k}^{(r)} z_{k}
$$

for our $r$-step fixed-point approximation to the exact solution

$$
\widetilde{u}=A_{n(F), n(Q)}^{\mathrm{INT}}(f, q)=\sum_{k=1}^{m} v_{k} z_{k} .
$$

Let us write

$$
u_{n(F), n(Q)}^{(r)}=A_{n(F), n(Q)}^{\mathrm{INT}-\mathrm{MOD}, r}(f, q),
$$

calling $A_{n(F), n(Q)}^{\mathrm{INT}, r \text {, } r}$ the modified interpolatory algorithm.

We now analyze the cost of computing $\widetilde{u}=A_{n(F), n(Q)}^{\mathrm{INT}}(f, q)$. We shall make the usual assumption, commonly made in information-based complexity theory, that arithmetic operations have unit cost and that one information operation of $f$ and $q$ have a fixed cost $\mathbf{c}_{d} \geq 1$. Now let

$$
\operatorname{cost}\left(\varepsilon, A_{\varepsilon, d}^{\mathrm{INT}}\right)=\inf \left\{\operatorname{cost}\left(A_{n(F), n(Q)}^{\mathrm{INT}}\right): e\left(A_{n(F), n(Q),}^{\mathrm{INT}}, S_{d}\right) \leq \varepsilon\right\}
$$

and

$\operatorname{cost}\left(\varepsilon, A_{\varepsilon, d}^{\mathrm{INT}-\mathrm{MOD}, r}\right)=\inf \left\{\operatorname{cost}\left(A_{n(F), n(Q)}^{\mathrm{INT}-\mathrm{MOD}, r}\right): e\left(A_{n(F), n(Q), r}^{\mathrm{INT}}, S_{d},\right) \leq \varepsilon\right\}$,

respectively, denote the minimal cost of using the interpolatory and modified interpolatory algorithms to find an $\varepsilon$-approximation of the Fredholm problem. That is, we minimize the cost by choosing proper parameters $n(F), n(Q)$ and $r$ of the modified interpolatory algorithm, and the parameters $n(F)$ and $n(Q)$ of the interpolatory algorithm.

As in [2], we have 
Proposition 3.2. For any $\varepsilon \in(0,1)$ and $d \in \mathbb{N}$, let $r$ in $(13)$ be given by

$$
r=\left\lceil\frac{\ln (2 /(1-M))+\ln (1 / \varepsilon)}{\ln (1 / M)}\right\rceil=\Theta\left(\ln \frac{1}{\varepsilon}\right) .
$$

Choose $n(F)$ and $n(Q)$ so that

$$
e\left(A_{n(F), n(Q)}^{\mathrm{INT}}\right) \leq \frac{1}{2} \varepsilon
$$

Then, using $r$ iterations of the fixed-point algorithm (13), we find that

$$
\operatorname{cost}\left(\varepsilon, A_{\varepsilon, d}^{\mathrm{INT}-\mathrm{MOD}, r}\right)=\mathbf{c}_{d} \cdot \Theta\left(n\left(\frac{1}{2} \varepsilon, A_{\varepsilon, d}^{\mathrm{INT}}\right) \ln \left(\frac{1}{\varepsilon}\right)\right)
$$

where the $\Theta$-factor is independent of $d$ and $\varepsilon$. Hence, if

$$
n\left(\frac{1}{2} \varepsilon, A_{\varepsilon, d}^{\mathrm{INT}}\right)=\mathscr{O}\left(n\left(\varepsilon, A_{\varepsilon, d}^{\mathrm{INT}}\right)\right)
$$

with $\mathscr{O}$-factor independent of $d$ and $\varepsilon$, then

$$
\operatorname{cost}\left(\varepsilon, A_{\varepsilon, d}^{\mathrm{INT}-\mathrm{MOD}, r}\right)=\mathbf{c}_{d} \cdot \Theta\left(n\left(\varepsilon, A_{\varepsilon, d}^{\mathrm{INT}}\right) \ln \left(\frac{1}{\varepsilon}\right)\right) .
$$

Hence, when (14) holds, the modified interpolatory algorithm is within a logarithmic factor of being optimal. Such is the case when the Fredholm problem (equivalently, the $L_{2}$-approximation problems for $F$ and $Q$ ) is strongly polynomially tractable or polynomially tractable. Obviously, the extra factor $\ln (1 / \varepsilon)$ does not change the exponents of strong polynomial or polynomial tractability.

\section{ENDNOTES}

1. In this paper, we are only concerned with such continuous linear information, rather than standard information (consisting of the values of the kernel or right-hand side at points in their domains).

2. By the $L_{2}$-approximation for $F$ (or for $Q$ ) we mean approximation of $f \in F_{d}$ (or $q \in Q_{2 d}$ ) in the $L_{2}$-norm for all $d$. 


\section{REFERENCES}

1. E. Novak and H. Woźniakowski, Tractability of multivariate problems, Volume 1: Linear information, EMS Tracts Math. 6, European Mathematical Society (EMS), Zürich, 2008.

2. A.G. Werschulz and H. Woźniakowski, Tractability of the Fredholm problem of the second kind, J. Integral Equations Applications 23 (2012), 413-461.

Department of Computer and Information Sciences, Fordham University, New York, Ny 10023 and Department of Computer Science, Columbia University, New York, NY 10027

Email address: agw@cs.columbia.edu

Department of Computer Science, Columbia University, New York, Ny 10027 and Institute of Applied Mathematics, University of Warsaw, Poland Email address: henryk@cs.columbia.edu 